\title{
Therapeutic effect of exogenous bone marrow-derived mesenchymal stem cell transplantation on silicosis via paracrine mechanisms in rats
}

\author{
MAN-MAN ZHAO ${ }^{1}$, JIAN-ZHONG CUI ${ }^{2}$, YING CUI $^{2}$, RAN LI $^{1}$, YAN-XIA TIAN $^{1}$, \\ SI-XIN SONG ${ }^{3}$, JUAN ZHANG ${ }^{1}$ and JUN-LING GAO ${ }^{1}$ \\ ${ }^{1}$ School of Basic Medical Science, Hebei United University; ${ }^{2}$ Tangshan Workers' Hospital, Tangshan, Hebei 063000; \\ ${ }^{3}$ Department of Surgery, Hebei Medical University, Shijiazhuang, Hebei 050017, P.R. China
}

Received November 27, 2012; Accepted April 9, 2013

DOI: $10.3892 / \mathrm{mmr} .2013 .1580$

\begin{abstract}
Silicosis is a well-known occupational disease, characterized by epithelial injury, fibroblast proliferation, expansion of the lung matrix and dyspnea. At present, no effective treatment methods for silicosis have been identified. The present study aimed to investigate the protective potential of exogenous bone marrow-derived mesenchymal stem cell (BMSC) transplantation on experimental silica-induced pulmonary fibrosis in rats and analyze the underlying paracrine mechanisms associated with its therapeutic effects. BMSCs were isolated, cultured and passaged from male Sprague-Dawley (SD) rat bone marrow. Third-generation BMSCs were identified by flow cytometry using FITC staining. Following the successful establishment of the silicosis model, exogenous BMSCs were infused into female adult SD rats via the tail vein. Lungs were evaluated using hematoxylin and eosin (H\&E) staining. The expression of interleukin-1 receptor antagonist (IL-1RA), interleukin-1 (IL-1) and tumor necrosis factor $\alpha$ (TNF- $\alpha$ ) protein was detected by immunohistochemistry and western blot analysis. Co-localization of sex determining region $\mathrm{Y}$ (SRY) and IL-1RA expression was determined by double-label immunofluorescence. The distribution of transplanted BMSCs was tracked by monitoring the expression of SRY in rats. Treatment with BMSCs was found to protect the lungs against injury and fibrosis by the suppression of upregulated IL-1 and TNF- $\alpha$ protein, via triggering IL-1RA secretion. This mechanism was hypothesized to be mediated by paracrine signaling.
\end{abstract}

Correspondence to: Professor Jun-Ling Gao, School of Basic Medical Science, Hebei United University, 57 South Jianshe Road, Tangshan, Hebei 063000, P.R. China

E-mail: junlinggao@yeah.net

Abbreviations: BMSCs, bone marrow-derived mesenchymal stem cells; IL-1, interleukin-1; IL-1RA, interleukin-1 receptor antagonist; TNF- $\alpha$, tumor necrosis factor $\alpha$; SRY, sex determining region $Y$

Key words: BMSCs, silicosis, IL-1, IL-1RA, TNF- $\alpha$, SRY
These results indicate that the release of IL-1RA from BMSCs via paracrine mechanisms significantly blocks the production and/or activity of IL-1 and TNF- $\alpha$. The present study provides an experimental basis for cellular therapy in silicosis.

\section{Introduction}

Silicosis is a significant global health issue, caused by long-term inhalation of high levels of dust containing excessive free silica during the production process. Pathological characteristics include silicotic nodule formation and pulmonary interstitial fibrosis (1). In addition, the incidence and prevalence of silicosis is markedly increasing and effective therapies are not currently available. Stem cell therapy represents a novel treatment modality associated with increasing therapeutic potential. Pluripotent adult stem cells located in the bone marrow are divided into two main distinct populations, hematopoietic and bone marrow-derived mesenchymal stem cells (BMSCs) (2). Over the last decade, BMSCs have been exploited as therapeutic vectors or tools for the treatment of a wide variety of diseases. Previous studies on cell transplantation demonstrated that the administration of BMSCs in animal models of injury exhibits protective effects following acute spinal cord (3), traumatic brain (4) or liver (5) injuries. In addition, a number of studies have revealed that exogenous delivery of BMSCs protects against a variety of pulmonary diseases, including acute lung injury $(6,7)$ and chronic lung disease (8). In addition, BMSC treatment has been identified to promote the repair of tissue structure and function following bleomycin (BLM)- (9), monocrotaline- (10) or lipopolysaccharide-induced (6) lung injury. Donor-derived BMSCs may target lung injuries, migrate to areas of damage and then differentiate into lung-specific cells (11), including type I and type II alveolar epithelial cells, endothelial cells, fibroblasts and bronchial epithelial cells, as shown in the rat model of bleomycin-induced pulmonary fibrosis (9), demonstrating the plasticity of BMSCs in this setting. These observations strengthen the hypothesis that BMSC therapy may be suitable for the treatment of pulmonary fibrosis.

At present, the majority of studies focus on the protective mechanisms of BMSCs that have been systemically trans- 
planted. The protective potential of BMSCs in tissue injury has been reported to be associated with the differentiation potential of engrafted BMSCs into specific-cell phenotypes (9), an increase in circulating levels of G-CSF and GM-CSF (11) and BMSC-driven immunomodulation caused by a shift in the Th1/Th2 balance (12). However, a newly emerging concept in tissue repair is associated with the hypothesis that BMSC efficacy is attributed, in part, to paracrine mechanisms $(7,13,14)$. Specifically, multiple cytokines, including vascular endothelial growth factor, stromal cell derived factor-1, insulin growth factor-1 and basic fibroblast growth factor, are released by BMSCs into conditioned medium in a paracrine fashion. These cytokines are associated with an anti-apoptotic effect of BMSCs in the ischemic myocardium (15). In addition, a large variety of factors have been reported to be secreted from BMSCs via a paracrine pathway, to induce growth and differentiation and prevent injured cells from apoptotic death (16). These mediators released from BMSCs exhibit numerous effects and exert protection on injured tissues. Collectively, these observations indicate that the efficacy of BMSCs may be explained by paracrine mechanisms. Notably, the current review highlights a considerable shift from BMSCs to BMSC-conditioned media (BMSC-CM) as an effective regenerative therapy. In addition, a growing number of studies are reporting that the delivery of concentrated BMSC-CM provides a significantly superior protection against diseases compared with the effects of BMSC administration $(17,18)$. Mechanisms of the effects of BMSC-CM also attribute to the production of a series of cytokines and chemokines, released from BMSCs via the paracrine pathway (19), demonstrating that BMSCs participate in injury repair via the secretion of specific soluble factors. These observations indicate that the therapeutic response of BMSCs is mediated by paracrine mechanisms. In addition, the efficacy of BMSC-CM contributes to an improved understanding of paracrine mechanisms.

Based on these observations, we hypothesize that exogenous BMSC administration protects against silicosis by two mechanisms. Firstly, by proliferation, homing and differentiation into specific lung cell types; and secondly, by paracrine mechanisms, involving the secretion of a wide range of soluble factors to improve pathological injury. Therefore, the main purpose of the present study was to evaluate the potential effects of BMSC engraftment on silica-induced pulmonary inflammation and fibrosis, and to determine whether BMSCs release IL1-RA into areas of lung injury via paracrine pathways to alleviate the early inflammatory response and the extent of fibrosis. In summary, results of this study are likely to lay the foundations for gene and stem cell therapy in silicosis in the future.

\section{Materials and methods}

Cell culture. BMSCs were generated from male Sprague-Dawley (SD) rats. Fresh bone marrow cells were collected by flushing the medullary cavity of rat femurs with Dulbecco's Modified Eagle's Medium (DMEM; Gibco-BRL, Carlsbad, CA, USA). After filtering, cells were centrifuged at 1,000 x $\mathrm{g}$ for $5 \mathrm{~min}$. Purified cells were dispersed in cell culture flasks (Corning Life Sciences, Tewksbury,MA,USA), grown in DMEM supplemented with $10 \%$ fetal calf serum (Gibco-BRL), $100 \mathrm{U} / \mathrm{ml}$ penicillin and $100 \mu \mathrm{g} / \mathrm{ml}$ streptomycin (Sigma-Aldrich, St. Louis, MO, USA) and then cultured at $37^{\circ} \mathrm{C}$ with $5 \% \mathrm{CO}_{2}$. Following $48 \mathrm{~h}$, non-adherent cells were removed and fresh media was added. Media was replaced every 3 days. Adhered cells were allowed to grow to $\sim 90 \%$ confluency and then trypsinized and reseeded. Passage 3 (P3) BMSCs were used for this experiment. The study was approved by the Ethics committee of The School of Basic Medical Sciences, Hebei United University, Tangshan, China.

Flow cytometry. P3 BMSCs were harvested by trypsinization (Gibco-BRL) and cells were fixed in neutralized 2\% paraformaldehyde solution for $30 \mathrm{~min}$. Fixed cells were washed three times with PBS and incubated with antibodies against the following antigens: CD19, CD34, CD44 and CD90 (all from Santa Cruz Biotechnology, Santa Cruz, CA, USA; 1:200) for $30 \mathrm{~min}$. Primary antibodies were directly conjugated with FITC. Flow cytometry was performed with a FACScan flow cytometer (BD Biosciences, Franklin Lakes, NJ, USA).

Animals and silicosis model. BMSC donor animals were five male adult SD rats, aged 3-5 weeks and weighing 100-120 g. BMSC recipient animals were 30 female adult SD rats, aged 6-8 weeks and weighing 200-220 g. All animals were provided by Vital River Laboratory Animal Technology Co., Ltd. (Beijing, China). The rat model of silicosis was induced by $1 \mathrm{ml}$ One-time infusing silica (Sigma-Aldrich) suspension $(5 \mathrm{~g} / \mathrm{l})$ using the non-exposed tracheal intubation. All rats were maintained in a room with a reversed 12-h light-dark cycle at constant temperature $\left(21^{\circ} \mathrm{C}\right)$ and humidity $(55 \%)$.

Groups and BMSC administration. BMSC recipient rats were randomly divided into three groups ( $\mathrm{n}=10 /$ group); the control, model and BMSC-treated groups. Rats in the control group were intratracheally injected with sterile saline. Following intratracheal administration of silica suspension, $1 \mathrm{ml}$ BMSC suspension $\left(3 \times 10^{6}\right.$ cells $\left./ \mathrm{ml}\right)$ was injected through a tail vein puncture. All rats in each group were sacrificed 14 days following surgery.

Histological analysis. Lung tissues were fixed in $4 \%$ paraformaldehyde solution for $24 \mathrm{~h}$, washed with running water for $2 \mathrm{~h}$, then dehydrated with gradient alcohol and embedded in paraffin following the standard histology procedure. Tissues were serially sectioned at a thickness of $5 \mu \mathrm{m}$. All sections were mounted on glass slides and then stained with hematoxylin and eosin (H\&E). Sections were observed and analyzed using an optical microscope.

Immunohistochemical analysis. Surgery was performed according to the manufacturer's instructions obtained from the SABC immunohistochemistry kit (Wuhan Boster Biological Technology,Ltd., Wuhan,China).Paraffinembeddedlung tissue sections $(5 \mu \mathrm{m})$ on poly-1-lysine-coated slides were heated for $30 \mathrm{~min}$ at $60^{\circ} \mathrm{C}$, dewaxed and rehydrated, followed by microwave antigen retrieval. Endogenous peroxidase was inactivated with $3 \% \mathrm{H}_{2} \mathrm{O}_{2}$ for $10 \mathrm{~min}$ at room temperature. Sections were incubated in 5\% BSA solution for $20 \mathrm{~min}$ to block nonspecific binding. Next, sections were incubated overnight at $4^{\circ} \mathrm{C}$ with rabbit anti-interleukin-1 receptor antagonist (IL-1RA), -interleukin-1 (IL-1) or -tumor necrosis factor- $\alpha$ (TNF- $\alpha$ ) polyclonal 
antibodies (Santa Cruz Biotechnology; 1:100), and then with horseradish peroxidase-conjugated anti-rabbit IgG antibodies for $30 \mathrm{~min}$. DAB was used to reveal the immunohistochemical reaction. PBS was substituted for the primary antibody as the negative control.

Western blot analysis. RIPA lysis buffer $(1 \mathrm{ml})$ was added to $100 \mathrm{mg}$ lung tissue and the mixture was homogenated at a low temperature $\left(4^{\circ} \mathrm{C}\right)$. Lysates from tissue samples were cleared by centrifugation at $10,000 \mathrm{x}$ g for $10 \mathrm{~min}$ at $4^{\circ} \mathrm{C}$. The protein concentration of samples was determined using BCA reagent (Beijing Solarbio Science \& Technology Co., Ltd., Beijing, China). Samples were subjected to sodium dodecyl sulfate polyacrylamide gel electrophoresis. Proteins were transferred onto PVDF membranes (Roche Diagnostics $\mathrm{GmbH}$, Mannheim, Germany). Blots were blocked with 5\% fat-free dry milk for $1 \mathrm{~h}$ at room temperature. Next, blots were incubated with the following primary antibodies overnight at $4^{\circ} \mathrm{C}$ : rabbit polyclonal anti-IL1-RA, -IL-1 and -TNF- $\alpha$ (1:400), mouse monoclonal anti- $\beta$-actin (Santa Cruz Biotechnology; 1:500). The blots were then incubated with alkaline phosphatase conjugated anti-rabbit IgG and anti-mouse IgG (Cell Signaling Technology, Inc., Danvers, MA, USA; 1:3,000) for $2 \mathrm{~h}$ at room temperature. Blots were developed with alkaline phosphatase color development kit (Beijing Solarbio Science \& Technology Co., Ltd., Beijing, China).

Immunofluorescence analyses. Lung tissues were fixed in $4 \%$ paraformaldehyde for $24 \mathrm{~h}$, submerged in $30 \%$ sucrose solution (0.1 M PBS, pH 7.4) until sinking to the bottom and then embedded in optimum cutting temperature compound (OCT). Frozen sections $(10 \mu \mathrm{m})$ were sliced with a microtome, treated with $0.4 \%$ Triton X-100 for $10 \mathrm{~min}$ and blocked in normal donkey serum for $1 \mathrm{~h}$. For double labeling, frozen sections were incubated with a mixture of goat anti-sex determining region Y (SRY; Santa Cruz Biotechnology; 1:200) and rabbit anti-IL-1RA (1:200) polyclonal antibodies overnight at $4^{\circ} \mathrm{C}$. On the following day, sections were incubated with a mixture of fluorescein-conjugated anti-rabbit $\mathrm{IgG}$ and anti-goat $\mathrm{IgG}$ (Santa Cruz Biotechnology; 1:1,000) for $2 \mathrm{~h}$ at $37^{\circ} \mathrm{C}$ in the dark. Images were captured using a laser scanning confocal microscope (Olympus FV1000; Olympus Inc., Center Valley, PA, USA). Primary antibodies were replaced with PBS in the negative control group. Additional immunofluorescence staining was performed in accordance with the described experimental procedure, to detect expression of SRY in the heart, liver, spleen, kidney and lung tissues of rats in the BMSC-treated group.

Statistical analysis. All experiments were repeated three times and similar results were obtained. Statistical analysis was performed using the SPSS 16.0 statistics software (SPSS, Inc., Chicago, IL, USA). Data are expressed as the mean \pm SE and the significance of the experimental results was determined using one-way ANOVA. $\mathrm{P}<0.05$ was considered to indicate a statistically significant difference.

\section{Results}

BMSC growth state and surface markers. Primary cells adhered within $48 \mathrm{~h}$ and proliferated in clone mode. Cell morphological characteristics included circles and polygons, nuclear-centered and occasional polygonal cells. BMSCs were purified by changing the medium several times and during this process, hematopoietic stem cells and other non-adherent growth cells were removed. Following 2 weeks, primary cells reaching $90 \%$ confluence were passaged. Passaged cells overcame the growth inhibition period and underwent accelerated growth. Following passaging, cell morphology was more uniform and consistent, forming mainly fibroblast-like flattened cells. The identity of the undifferentiated BMSCs was confirmed by detecting specific cell surface markers. Flow cytometry analysis of P3 BMSCs indicated that BMSCs were CD44- and CD90-positive and CD19- and CD34-negative.

Confirmation of construction of the silicosis model. Successful construction of the rat model of silicosis was confirmed by H\&E staining. As demonstrated in Fig. 1A, alveolitis change, silicosis nodule formation and collagen deposition were observed in the model group, confirming successful construction of the model. By contrast, clear alveolar structures and walls were present, and inflammatory cell infiltration was not observed in the control group. However, following transplantation of the BMSCs, pathological changes associated with silicosis were significantly reduced. These results indicate that the silicosis model was successfully constructed and delivery of BMSCs exerted a significant protective effect. In addition, the majority of IL-1RA released by transplanted BMSCs appeared to be mediated in a paracrine manner.

BMSCs upregulate IL-1RA to suppress IL-1 and TNF- $\alpha$ expression. Immunohistochemical analysis was used to examine the localization of IL-1RA (Fig. 1B), IL-1 (Fig. 1C) and TNF- $\alpha$ (Fig. 1D) protein expression. Brown particles observed in cells were considered as positive results. Brown particles appeared in macrophages, alveolar epithelial cells and inflammatory cells. Western blot (Fig. 2) and immunohistochemical analysis revealed trends in IL-1RA, IL-1 and TNF- $\alpha$ expression. IL-1RA, IL-1 and TNF- $\alpha$ proteins were expressed at low levels in the control group. Their expression was increased in the model group compared with the control group. Following treatment with BMSCs, IL-1RA protein expression was markedly upregulated compared with the model group. Furthermore, IL-1 and TNF- $\alpha$ protein expression levels were significantly decreased compared with the model group; however, these remained higher than levels in the control group. Overall, these findings indicate that the release of IL-1RA blocks the production and/or activity of IL-1 and TNF- $\alpha$ proteins.

BMSCs release IL-1RA into injured lungs. Double immunofluorescence staining was performed to investigate the co-localization of SRY and IL-1RA expression. As shown in Fig. 3A, SRY was stained with goat anti-SRY and fluorescently labeled (green) secondary antibodies. IL-1RA was stained with rabbit anti-IL1RA and fluorescently labeled (red) secondary antibodies. Fluorescence was observed under a laser scanning confocal microscope; yellow fluorescence indicated co-localized expression in merged images. These results suggest that the majority of IL-1RA was released by transplanted BMSCs in a paracrine manner. 


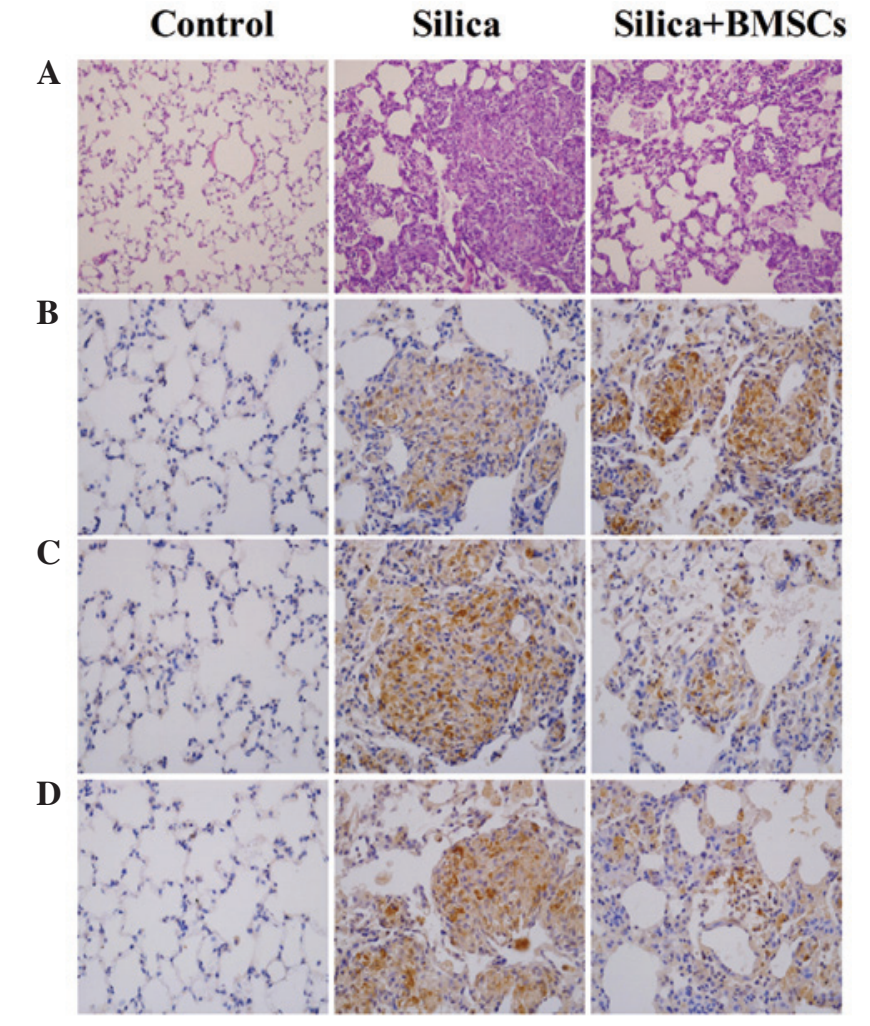

Figure 1. Morphological changes in lung tissues at day 14. Representative images of lungs stained with H\&E. (A) Images revealed the infiltration of inflammatory cells and formation of silicon nodules, confirming that the rat model of silicosis was successfully constructed (magnification, x200). Immunohistochemical staining of (B) IL-1RA, (C) IL-1 and (D) TNF- $\alpha$ proteins in the rat lung. Brown particles represent positive cells (magnification, x400). BMSCs, bone marrow-derived mesenchymal stem cells; $\mathrm{H} \& \mathrm{E}$, hematoxylin and eosin; IL-1RA, interleukin-1 receptor antagonist; IL-1, interleukin-1; TNF- $\alpha$, tumor necrosis factor- $\alpha$.

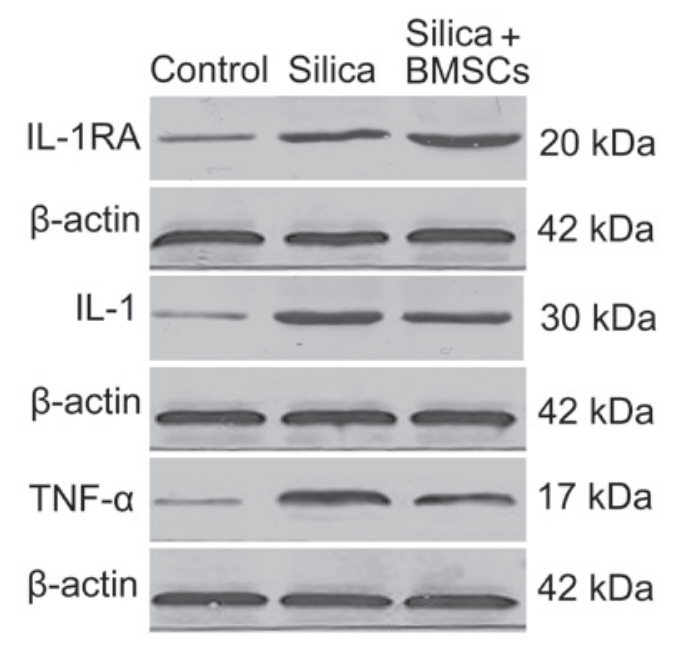

Figure 2. Western blot analysis of IL-1RA, IL-1 and TNF- $\alpha$ protein expression, revealing marked changes in protein expression for the control, silica and silica + BMSCs groups. IL-1RA, interleukin-1 receptor antagonist; IL-1, interleukin-1; TNF- $\alpha$, tumor necrosis factor- $\alpha$; BMSCs, bone marrow-derived mesenchymal stem cells.

BMSC aggregation in injured lungs. SRY expression was determined by immunofluorescent staining 14 days after administration of BMSCs. SRY protein was stained using
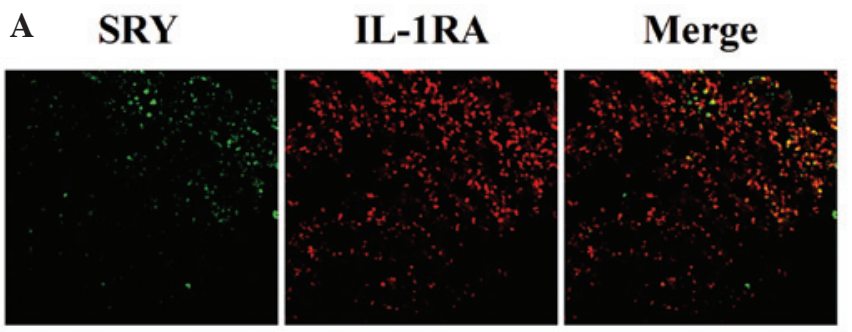

B

heart

liver

spleen
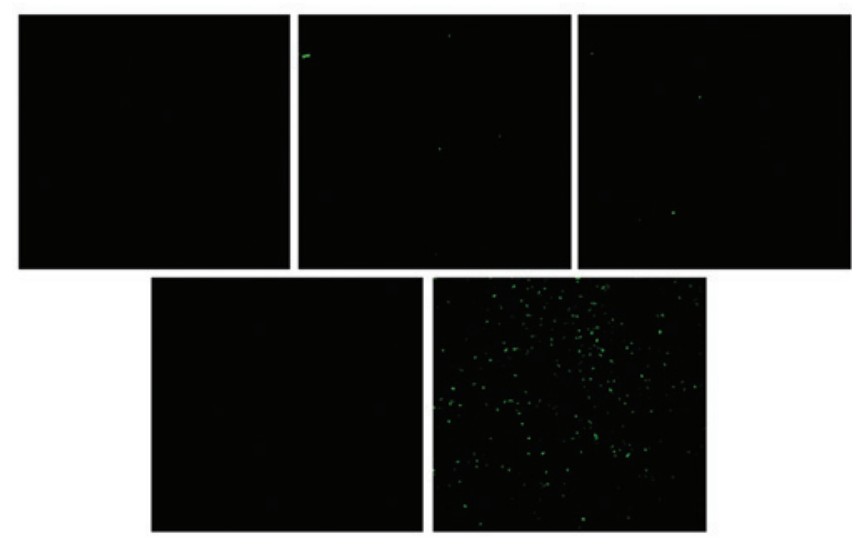

kidney

lung

Figure 3. (A) Co-localization of SRY and IL-1RA was determined by immunofluorescent staining (magnification, x400). (B) Distribution of SRY in the heart, liver, spleen, kidney and lung tissues of BMSC-treated rats. All microphotographs were visualized by confocal laser scanning microscopy (magnification, x200). IL-1RA, interleukin-1 receptor antagonist; SRY, sex determining region Y; BMSC, bone marrow-derived mesenchymal stem cell.

goat anti-SRY and secondary antibodies labeled with green fluorescence. As revealed in Fig. 3B, BMSCs expressing SRY (green) were identified in lung tissues, but not in tissues of the heart, liver, spleen and kidneys in BMSC-treated rats. These observations indicated that injured tissues were 'sensed' by engrafted BMSCs. Subsequently, BMSCs homed and localized to the injured lung tissue, exerting important biological functions in damaged areas.

\section{Discussion}

Silicosis is associated with significant levels of morbidity and mortality. In addition, the development of fibrosis during silicosis is associated with a complex pathogenesis (1). Therefore, treatment strategies require a multi-angle, multi-link and multi-targeted approach. To date, no efficient clinical treatments are available, and the identification of safe and effective therapeutic strategies for this pathology is of considerable urgency. Over previous years, we have been committed to the study of the prevention and treatment of silicosis. Exogenous BMSC transplantation therapies may represent a novel therapeutic strategy for lung diseases currently lacking efficient treatments and are likely to be important in silicosis clinical care in the future.

The primary objective of the present study was to verify our hypothesis that the protective effects of BMSCs on silicosis injury are mediated, in part, by the secretion of IL-1RA 
in a paracrine manner. It was previously reported that IL-1RA exhibits a wide range of biological functions, including competitively inhibiting IL-1 activity (20) and blocking the production and/or activity of IL-1 and TNF- $\alpha$ (21). In the current study, BMSCs were selected as a novel method for silicosis treatment due to their capacity to undergo self-renewal, proliferation and differentiation into multiple cell lineages. More importantly, BMSCs are associated with low immunogenicity and no immune rejection, enabling BMSC therapies to be applied for daily clinical applications. As a result of these advantageous properties, BMSCs have been applied in a number of disease types in animals and human patients. However, the mechanisms responsible for their therapeutic effect remain unknown.

In the current study, BMSCs were cultured and passaged using the cell adherent method (22) and P3 BMSCs were used in the experiments. Cells were identified via flow cytometry analysis and results confirmed that the BMSCs expressed characteristic surface markers, CD44 and CD90, and were CD19- and CD34 negative, indicating the multilineage potential of BMSCs. Distribution of BMSCs in recipient rats was determined by immunofluorescence staining. SRY (green) was only observed in the lung tissue, indicating that BMSCs were able to 'sense' the environment and respond according to the requirements of the organism for survival (23). Following this, BMSCs target the site of lung injury, assume specific and distinct lung cell phenotypes and regulate inflammatory responses, to promote structural and functional repair (9). Finally, intravenous administration of BMSCs was revealed to exhibit a marked therapeutic potential for silicosis in rats. These observations indicate that injured lung tissue produces chemokines responsible for the mobilization and homing of BMSCs towards the site of damage. In addition, the fate of engrafted BMSCs in vivo is largely regulated by the microenvironment.

Based on these observations, the differentiation of BMSCs into specific cell phenotypes appears to be crucial in injury repair. However, differentiation does not fully account for the strong therapeutic response observed, indicating that other specific mechanisms may be involved in the repair of lung injury by BMSCs. Notably, an important observation of the present study was that the efficiency of BMSCs in injury repair was more likely to be due to paracrine effects. It was previously reported that BMSCs secrete a wide array of growth factors, cytokines and immunomodulatory factors (24) and release numerous angiogenic, anti-apoptotic and mitogenic factors (25), which may afford protection to injured tissue. However, little is known with regard to variations in the secretion of paracrine factors between various subpopulations of BMSCs. Substrate-dependent paracrine signaling has been demonstrated between subpopulations of BMSCs, which may affect their formation or perhaps malformation (26). In addition, Bakondi et al (18) examined typical, CD133-derived and p75LNGFR-derived BMSCs, observing different secretion responses in each population in terms of the levels of secreted growth factors and cytokines when exposed to a hypoxic environment. Consistent with these observations, in the current study, intravenous administration of BMSCs as a novel method of treatment for silicosis protected lungs not only through multi-lineage differentiation mechanisms, but also through paracrine mechanisms.
In this study, the tracking of BMSCs was essential for evaluation of their early migration and distribution patterns in rats. Therefore, BMSCs isolated from male rats were injected into female rats in vivo. Next, the expression of SRY was detected by laser scanning confocal microscopy to track BMSC survival and further co-localization with paracrine factors. The results of H\&E staining revealed the marked infiltration of inflammatory cells and silicotic nodule formation in the model group, indicating that the rat model of silicosis was constructed successfully. Following administration of BMSCs, these pathological changes were reduced, indicating that BMSCs significantly reduced inflammation and the extent of fibrosis. Prevention of the inflammatory response and inhibition of collagen accumulation and matrix metalloproteinase activation by BMSCs has also been demonstrated in BLM-induced lung injury in mice (9). In this study, administration of rat BMSCs $24 \mathrm{~h}$ following silica treatment was observed to result in a significant reduction in IL-1 and TNF- $\alpha$ levels, explained, in part, by an increase in IL-1RA expression. In addition, the fluorescent double-labeled co-localization of SRY (green) and IL-1RA (red) expression was observed in the BMSC-treated group. Co-localization of SRY and IL-1RA appeared yellow following the merging of images. Consistent with our hypothesis that BMSCs may secrete IL-1RA into damaged lung tissue via a paracrine pathway, Ortiz et al (21) reported that the expression and secretion of IL-1RA is restricted to a unique subpopulation of BMSCs. In addition, a previous study by Mei et al (27) demonstrated that human BMSCs possessed direct antimicrobial activity, which was mediated, in part, by the secretion of cathelicidin hCAP-18/LL-37, improving survival and enhancing bacterial clearance. More specifically, results of immunohistochemistry and western blot analysis indicated that IL-1RA released by BMSCs not only antagonized the function of IL-1, but also blocked the release of TNF- $\alpha$ from activated macrophages. IL-1 and TNF- $\alpha$, two fundamental pro-inflammatory cytokines in the lung, are vital in the pathogenic process of pulmonary fibrosis. Inflammatory responses in silicosis are mediated by the release of proinflammatory cytokines, IL-1 and TNF- $\alpha$, from activated macrophages and other leukocytes (28). IL-1 and TNF- $\alpha$ also directly or indirectly stimulate proliferation of endothelial cells and fibroblasts, extracellular matrix deposition and collagen synthesis (29). Finally, these cytokines promote the formation and development of pulmonary fibrosis. However, IL-1RA, a protein present in normal conditions, is known to reduce the degree of pulmonary fibrosis (30). Previous studies have reported that injection of IL-1RA intraperitoneally by implantation of a permeability micropump device revealed a marked protective potential from BLM- or silicon dioxide-induced pulmonary fibrosis (31), indicating that IL-1RA may block lung inflammation and fibrosis induced by exposure to silica. These studies provided a realistic and viable basis for the present study.

In conclusion, results of the current study demonstrate that BMSCs have a comprehensive biological effect on silicosis. Secretion of IL1-RN by BMSCs in a paracrine manner protected rats from silica-induced lung injury by blocking the production and/or activity of IL-1 and TNF- $\alpha$. This may perform an anti-inflammatory role in early stages of the disease and an antifibrotic role at later stages in silicosis rats. 
In short, BMSCs exhibit a potential beneficial effect on silicosis through paracrine mechanisms. This study may provide valuable insight into the prevention and treatment of silicosis and present novel prospects for cell therapy.

\section{Acknowledgements}

The present study was supported by grants from the Science and Technology Support Key Funding Project of Hebei Province (no. 09276191D), the State Production Safety Supervision Administration Science and Technology Development Funding Project (no. 06-549) and the Hebei Province Science and Technology Support for Major Projects (no. 13277709D).

\section{References}

1. Rimal B, Greenberg AK and Rom WN: Basic pathogenetic mechanisms in silicosis: current understanding. Curr Opin Pulm Med 11: 169-173, 2005.

2. Herzog EL, Chai L and Krause DS: Plasticity of marrow-derived stem cells. Blood 102: 3483-3493, 2003.

3. Nishida H, Nakayama M, Tanaka H, et al: Safety of autologous bone marrow stromal cell transplantation in dogs with acute spinal cord injury. Vet Surg 41: 437-442, 2012.

4. Osanai T, Kuroda S, Sugiyama T, et al: Therapeutic effects of intra-arterial delivery of bone marrow stromal cells in traumatic brain injury of rats - in vivo cell tracking study by near-infrared fluorescence imaging. Neurosurgery 70: 435-444, 2012.

5. Zhao L, Feng Z, Hu B, Chi X and Jiao S: Ex vivo-expanded bone marrow mesenchymal stem cells facilitate recovery from chemically induced acute liver damage. Hepatogastroenterology 59 2389-2394, 2012.

6. Islam MN, Das SR, Emin MT, et al: Mitochondrial transfer from bone-marrow-derived stromal cells to pulmonary alveoli protects against acute lung injury. Nat Med 18: 759-765, 2012.

7. Luan Y, Zhang X, Kong F, Cheng GH, Qi TG and Zhang ZH Mesenchymal stem cell prevention of vascular remodeling in high flow-induced pulmonary hypertension through a paracrine mechanism. Int Immunopharmacol 14: 432-437, 2012.

8. Aslam M, Baveja R, Liang OD, et al: Bone marrow stromal cells attenuate lung injury in a murine model of neonatal chronic lung disease. Am J Respir Crit Care Med 180: 1122-1130, 2009.

9. Ortiz LA, Gambelli F, McBride C, et al: Mesenchymal stem cell engraftment in lung is enhanced in response to bleomycin exposure and ameliorates its fibrotic effects. Proc Natl Acad Sci USA 100: 8407-8411, 2003

10. Luan Y, Zhang ZH, Wei DE, et al: Implantation of mesenchymal stem cells improves right ventricular impairments caused by experimental pulmonary hypertension. Am J Med Sci 343: 402-406, 2012

11. Rojas M, Xu J, Woods CR, et al: Bone marrow-derived mesenchymal stem cells in repair of the injured lung. Am J Respir Cell Mol Biol 33: 145-152, 2005.

12. Nemeth K, Keane-Myers A, Brown JM, et al: Bone marrow stromal cells use TGF-beta to suppress allergic responses in a mouse model of ragweed-induced asthma. Proc Natl Acad Sci USA 107: 5652-5657, 2010.

13. Wang PP, Xie DY, Liang XJ, et al: HGF and direct mesenchymal stem cells contact synergize to inhibit hepatic stellate cells activation through TLR4/NF-kB pathway. PLoS One 7: e43408, 2012.
14. Jin P, Zhang X, Wu Y, et al: Streptozotocin-induced diabetic rat-derived bone marrow mesenchymal stem cells have impaired abilities in proliferation, paracrine, antiapoptosis, and myogenic differentiation. Transplant Proc 42: 2745-2752, 2010.

15. Xu M, Uemura R, Dai Y, Wang Y, Pasha Z and Ashraf M: In vitro and in vivo effects of bone marrow stem cells on cardiac structure and function. J Mol Cell Cardiol 42: 441-448, 2007.

16. Mezey E: The therapeutic potential of bone marrow-derived stromal cells. J Cell Biochem 112: 2683-2687, 2011.

17. Reis LA,BorgesFT,Simões MJ,Borges AA,Sinigaglia-CoimbraR and Schor N: Bone marrow-derived mesenchymal stem cells repaired but did not prevent gentamicin-induced acute kidney injury through paracrine effects in rats. PLoS One 7: e44092, 2012.

18. Bakondi B, Shimada IS, Perry A, et al: CD133 identifies a human bone marrow stem/progenitor cell sub-population with a repertoire of secreted factors that protect against stroke. Mol Ther 17: 1938-1947, 2009.

19. Burdon TJ, Paul A, Noiseux N, Prakash S and Shum-Tim D: Bone marrow stem cell derived paracrine factors for regenerative medicine: current perspectives and therapeutic potential. Bone Marrow Res 2011: Dec 62010 (Epub ahead of print).

20. Arend WP: Interleukin 1 receptor antagonist. A new member of the interleukin 1 family. J Clin Invest 88: 1445-1451, 1991.

21. Ortiz LA, Dutreil M, Fattman C, et al: Interleukin 1 receptor antagonist mediates the antiinflammatory and antifibrotic effect of mesenchymal stem cells during lung injury. Proc Natl Acad Sci USA 104: 11002-11007, 2007.

22. Smajilagić A, Aljičević M, Redžić A, Filipović S and Lagumdžija A: Rat bone marrow stem cells isolation and culture as a bone formative experimental system. Bosn J Basic Med Sci 13: 27-30, 2013.

23. Abkowitz JL, Robinson AE, Kale S, Long MW and Chen J: Mobilization of hematopoietic stem cells during homeostasis and after cytokine exposure. Blood 102: 1249-1253, 2003.

24. Kinnaird T, Stabile E, Burnett MS, et al: Local delivery of marrow-derived stromal cells augments collateral perfusion through paracrine mechanisms. Circulation 109: 1543-1549, 2004.

25. Nagaya N, Kangawa K, Itoh T, et al: Transplantation of mesenchymal stem cells improves cardiac function in a rat model of dilated cardiomyopathy. Circulation 112: 1128-1135, 2005.

26. Sharma RI and Snedeker JG: Paracrine interactions between mesenchymal stem cells affect substrate driven differentiation toward tendon and bone phenotypes. PLoS One 7: e31504, 2012.

27. Krasnodembskaya A, Song Y, Fang X, et al: Antibacterial effect of human mesenchymal stem cells is mediated in part from secretion of the antimicrobial peptide LL-37. Stem Cells 28: 2229-2238, 2010.

28. Zhang $\mathrm{K}$ and Phan SH: Cytokines and pulmonary fibrosis. Biol Signals 5: 232-239, 1996.

29. Kolb M, Margetts PJ, Anthony DC, Pitossi F and Gauldie J: Transient expression of IL-1beta induces acute lung injury and chronic repair leading to pulmonary fibrosis. J Clin Invest 107: 1529-1536, 2001

30. Zhang Y, Lee TC, Guillemin B, Yu MC and Rom WN: Enhanced IL-1 beta and tumor necrosis factor-alpha release and messenger RNA expression in macrophages from idiopathic pulmonary fibrosis or after asbestos exposure. J Immunol 150: 4188-4196, 1993.

31. Piguet PF, Vesin C, Grau GE and Thompson RC: Interleukin 1 receptor antagonist (IL-1ra) prevents or cures pulmonary fibrosis elicited in mice by bleomycin or silica. Cytokine 5: 57-61, 1993. 